\title{
Necessity of Education and Training of the Healthcare Staffs Performing Sterilization and Disinfection of Surgical Instruments
}

\author{
Sifat Uz Zaman ${ }^{1 \& 2 *}$, Israt Sadia ${ }^{3}$, Farha Rahman ${ }^{4}$, Rayhana Sharmin ${ }^{5}$, Samina Haque ${ }^{6}$, and Aklima \\ Ferdoush $^{7}$ \\ ${ }^{1}$ Dept. of Microbiology, Jahangirnagar University, Dhaka, Bangladesh; ${ }^{2}$ Dept. of Infection Prevention and Control, Medlife \\ Healthcare Limited, Bangladesh; ${ }^{3}$ Dept. of Infection Control, Labaid Cardiac Hospital, Bangladesh; ${ }^{4}$ Dept.of Microbiology, \\ Bangladesh Medical College, Bangladesh; ${ }^{5}$ Dept. of Pharmacology, Uttara Adhunik Medical College, Bangladesh; ${ }^{6}$ Dept. of \\ Psychiatry, Bangladesh Psychiatric Care Ltd, Bangladesh; and ${ }^{7}$ Dept. of Medicine, Z.H Sikder Women Medical College \& \\ Hospital, Bangladesh. \\ *Correspondence: sifat.zaman9@gmail.com (Sifat Uz Zaman, Assistant Manager \& Head, Dept. of Infection Prevention and \\ Control, Medlife Healthcare Limited, Dhaka, Bangladesh).
}

\section{ABSTRACT}

The objective of this study is to evaluate the requirements of education among the healthcare staffs that perform sterilization and disinfection of surgical instruments in the hospitals. The study was done from $3^{\text {rd }}$ July to $5^{\text {th }}$ August, 2020 in a super specialized hospital in Dhaka, Bangladesh. In the present study, the total sample was 73 which included the nurses and technicians and these people were involved in infection control procedures of the hospitals. Among them, 39 were nurses who completed their diploma or B.Sc in nursing, and 34 were technicians who do not have any degree in medical science. A close-ended questionnaire survey was administered. All the 73 staffs were invited individually to participate in this cross-sectional survey. Confidentiality of the responses was assured to the participants. The educated healthcare staffs had more positive attitude than the uneducated staffs. All the educated staffs had a positive attitude on having a centralized sterilization procedure, but the uneducated staffs had only $58.52 \%$ positive attitude. $100 \%$ of the staffs who were educated had the awareness of different sterilization and disinfection methods and their harmful effects, but this result was $73.52 \%$ in uneducated staffs. Almost half of the population did not have an awareness of post sterilization management of surgical instruments. The percentage of positive responses in using different types of sterilization monitoring tools was not satisfactory as few people were not aware of it and the result was $87.17 \%$. But a very poor result had been detected with the same question from the uneducated staffs which was $55.88 \%$. Similar type of result had also been detected in the attitude level of using different types of disinfectants for cleaning surgical instruments. The positive response was $94.87 \%$ in educated staffs whereas $64.70 \%$ in uneducated staffs. The uneducated healthcare staffs need to be focused more as we saw there is a huge gap in the positive awareness and attitude level from the above result.

Keywords: CSSD, Surgical site infection, Training, HAIs, Healthcare, Sterilization, and Disinfection.

\section{INTRODUCTION:}

Surgical site infections, pneumonia, lower respiratory tract infection, UTI and sepsis are the most commonly occurring infections in all over the world (Kaushal et UniversePG I www.universepg.com $a l .$, 2015). Among all the patients who were admitted in hospital $8.7 \%$ had HAIs (Healthcare Associated Infections) showed by a report of WHO (CDC, 2021). Failure in sterilization considered the primary reason 
for the infections in clinical sites and hence many reports have been found about HAIs worldwide due to improper sterilization (Rutala et al., 2016).

Sterilization is such a process of killing or destroying all kinds of microbes including spores which is neither easy to achieve nor easy to prove. Disinfection is killing or destruction of various organisms with the help of chemical or other solution and this procedure is considered to be less fatal than sterilization (Damani, 2019). Sterilizing equipment is very important for the patients who come in exposure with surgical instruments, syringes, bandages and gauges all the time. Ensuring the safety for the patients, all the medical equipment and consumables must be clean, disinfected and sterilized. All the microorganisms which are pathogenic should be completely destroyed to minimize HAIs (Shahen et al., 2019). HAIs are caused due to many reasons like contaminated products, sterilization failure of surgical and other instruments, breach of sterility during procedures, inappropriate hygiene of the staffs involved in the procedures and contaminated environment, etc.

Invasive and non-invasive medical devices safety cannot be achieved if disinfection and sterilization are not properly done. Principal criteria of international patient safety goal are prevention of HAI. Controlling and prevention of HAIs are a big challenge for the physicians. To cut back on the threat of HAIs, improvement in the application of sterilization and disinfection in hospital settings is necessary (Zaman et al., 2021). Protocols and procedures for cleaning, disinfection and sterilization apply to anyone reprocessing medical equipment/devices in the health care organization. Mandatory evaluation by the practitioners are necessary to evaluate, establish and update the settings and by their continuous supervision protocols and control strategies the infection prevention rate can be improved (RCDSO, 2012).
To perform these activities, professionally educated and trained healthcare staffs are required in hospital settings. Continual learning with practical implication must be ensured for all the staffs that provide healthcare services. This study is done to assess the requirements of education intended for the healthcare staffs that perform sterilization and disinfection of surgical instruments in the hospitals.

\section{METHODOLOGY:}

\section{Study area and population}

The study was done from $3^{\text {rd }}$ July to $5^{\text {th }}$ August, 2020 in a super specialized hospital in Dhaka. Total 73 responses were collected in this study which included the nurses and technicians who were involved in sterilization and disinfection procedure of surgical instruments. Among them, 39 were nurses who completed their diploma or B.Sc in nursing and 34 were technicians and these technicians were not qualified by any degree of medical science.

\section{Data collection}

A close-ended questionnaire survey was administered. The format of the questionnaire was in English. The information on demographic variables such as sex, age, educational qualification of the participants was wrapped up by the questionnaire. The questionnaire also included the details to discover the awareness and attitude level on sterilization and disinfection practice of both educated and uneducated healthcare staffs. All the 73 staffs were requested independently to take a part in this cross-sectional survey. It was assured to the participants about the confidentiality of their responses.

\section{Institutional clearance}

Institutional clearance was granted from the hospital before starting the questionnaire survey.

\section{Questionnaire:}

\begin{tabular}{|c|l|c|c|}
\hline No & \multicolumn{1}{|c|}{ Awareness and Attitude } & Answer & Code \\
\hline Q1 & Do you feel centralized sterilization area is necessary in hospital? & Yes $=1, \mathrm{No}=2$ & \\
\hline Q2 & Do you have any idea about various sterilization methods and their harmful effects? & $\mathrm{Yes}=1, \mathrm{No}=2$ & $\mathrm{Yes}=1, \mathrm{No}=2$ \\
\hline Q3 & Do you have any knowledge about post sterilization handling of surgical items? & $\mathrm{Yes}=1, \mathrm{No}=2$ \\
\hline Q4 & $\begin{array}{l}\text { Do you know that transmission of infections can happen if appropriate hygienic } \\
\text { precautions are not considered? }\end{array}$ & $\mathrm{Yes}=1, \mathrm{No}=2$ & \\
\hline Q5 & Should health care workers handle sterilization packaging materials? & & \\
\hline
\end{tabular}




\begin{tabular}{|c|l|c|c|}
\hline Q6 & $\begin{array}{l}\text { Should health care workers always put on proper personal protective attire while } \\
\text { disinfection and sterilization procedure? }\end{array}$ & Yes=1, No=2 & \\
\hline Q7 & $\begin{array}{l}\text { Before and after sterilization and disinfection of surgical instruments is it necessary to } \\
\text { perform hand hygiene? }\end{array}$ & Yes=1, No=2 & \\
\hline Q8 & Should health care workers use various methods of sterilization monitoring tools? & Yes=1, No=2 & \\
\hline Q9 & $\begin{array}{l}\text { Should nurses and technicians use distinct methods of disinfectants for processing } \\
\text { surgical instruments? }\end{array}$ & Yes=1, No=2 & \\
\hline Q10 & $\begin{array}{l}\text { Should medically care providers always follow proper sterilization and disinfection } \\
\text { guidelines or policy? }\end{array}$ & Yes=1, No=2 & Yes=1, No=2 \\
\hline Q11 & $\begin{array}{l}\text { Is it necessary to organize regular basis trainings and workshops for health staffs on } \\
\text { sanitization protocols and latest innovations? }\end{array}$ & Y \\
\hline
\end{tabular}

\section{RESULTSAND DISCUSSION:}

\section{Fundamental profile of health care workers among study population $(n=73)$}

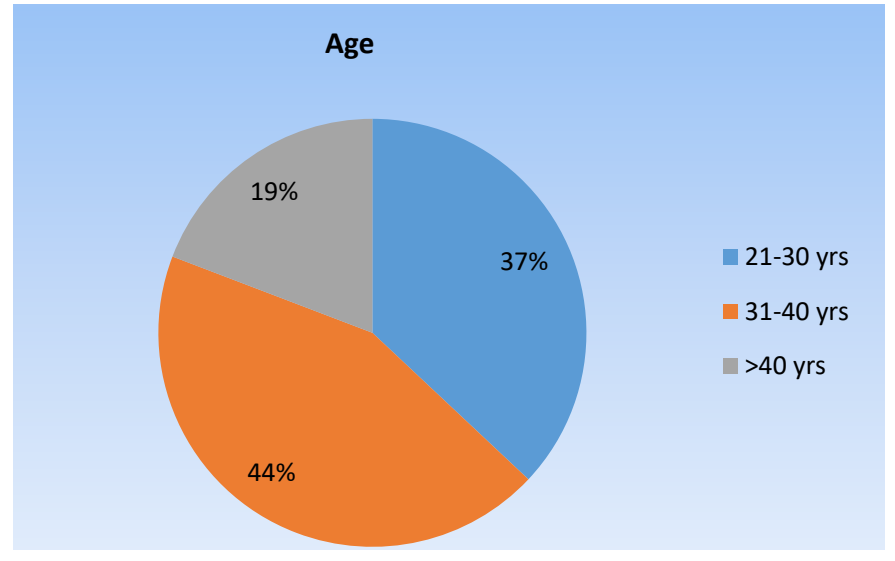

Fig 1: Age distribution.

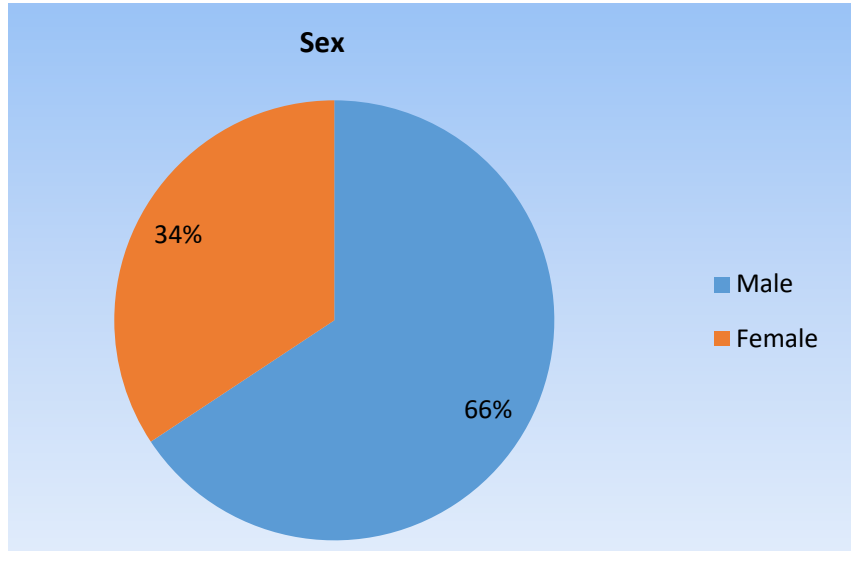

Fig 2: Gender distribution.

The percentages of positive response in awareness and attitudes of both educated and uneducated healthcare staffs concerning sterilization and disinfection are given below:

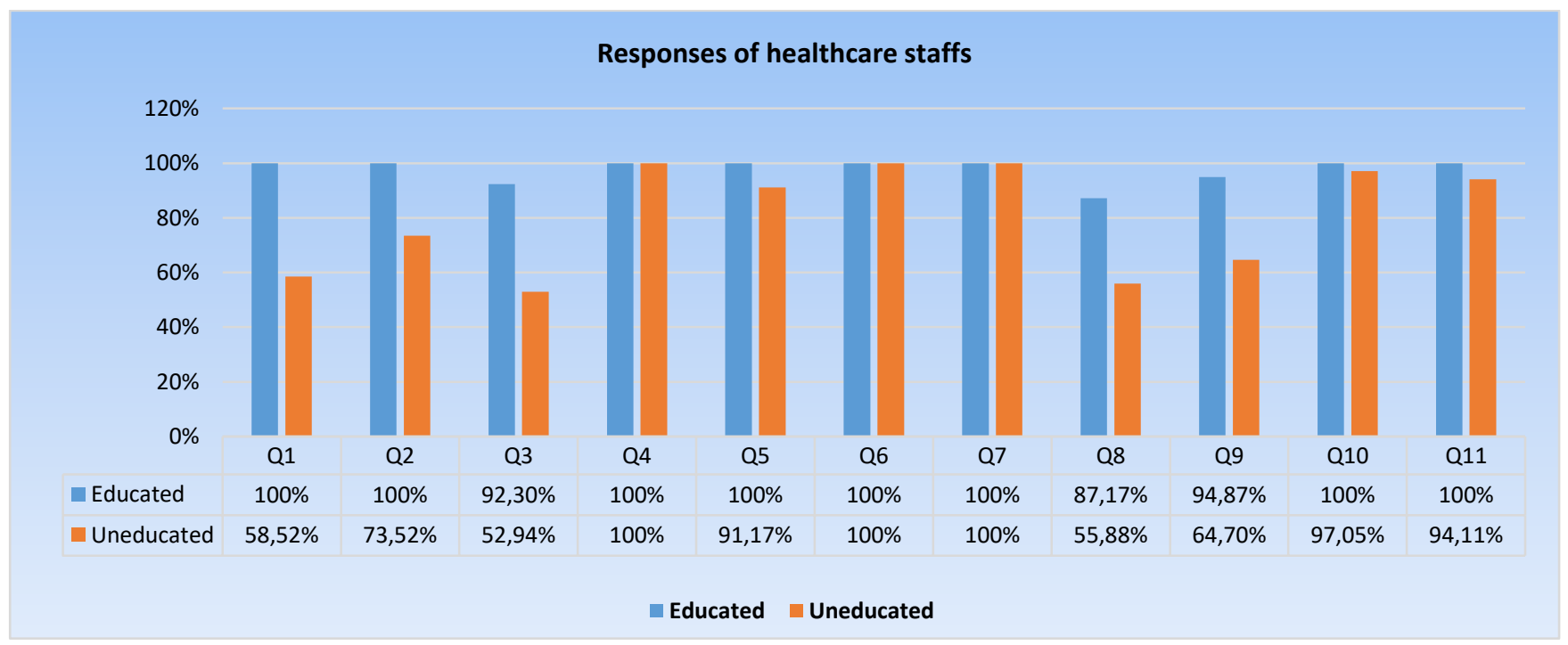

Fig 3: Positive responses of healthcare staffs (both educated and uneducated).

The necessity of education is very much clear when the above results are considered. We can comprehend UniversePG I www.universepg.com by the current study that educated healthcare staffs had more positive attitude than the uneducated staffs. All 
the educated staffs had a positive attitude on having a centralized sterilization facility but the uneducated staffs had only $58.52 \%$ positive attitude. $100 \%$ of the staffs who were educated had the knowledge of different sterilization and disinfection methods and their harmful effects but this result was $73.52 \%$ in uneducated staffs.

Almost half of the responding health care workers did not have the awareness of post sterilization handling of surgical instruments according to our study. The percentage of positive response in using various methods of sterilization monitoring tools was not satisfactory as few people were not aware of it; the result was $87.17 \%$ in educated population. Whereas a very poor result had been detected with the similar question from the uneducated staffs, which was $55.88 \%$. Similar type of result was also detected in the attitude level of using distinct methods of disinfectants for processing surgical instruments. The positive response was $94.87 \%$ in educated staffs whereas $64.70 \%$ in uneducated staffs. So, it was very clear that there was a huge gap in awareness and attitude level between the educated and uneducated staffs, this practice brings about great harm to the sick people who would be taken service from the uneducated staffs. The value of education in sterile processing was indicated in a study done by (Ahmed et al., 2017).

Dispersal of the nurse's opinion in percentage regarding the central sterile supply department services were very much praiseworthy, which corroborates the value of learning and practical implication in sterile processing (Ahmed et al., 2017). A study done by (Zaman et al., 2021) showed both home and abroad training facilities should be carried out for the healthcare staffs to enrich themselves. Hence, there is no alternative of knowledge to provide quality service to the sick people by the healthcare staffs (Alabrah et al., 2021; Zaman et al., 2021).

\section{CONCLUSION:}

Continuous education and training program is necessary for both the educated and uneducated healthcare staffs for raising awareness in sterilization and disinfection practice. Education and training for the health care staffs must be conducted by the hospital infection prevention and control team/officer regarding proper

UniversePG I www.universepg.com reprocessing of surgical instruments, at least once per month. The uneducated healthcare staffs need to be focused more as it is perceived that there is a huge gap in the positive awareness and attitude level from the above result. Therefore, educational programs and continuous training programs should be the main factors to overcome the problem.

\section{ACKNOWLEDGEMENT:}

We would like to thank Mr. M. Kamal Uddin, Managing Director, Medlife Healthcare Limited for his assistance and tremendous support throughout the research work.

\section{CONFLICTS OF INTEREST:}

This clear declaration from authors that there are no conflicts with due respect to the publication of this article.

\section{REFERENCES:}

1) Alabrah PW, Allagoa DO, and Eguvbe AO. (2021). Assessment of healthcare practitioners' insight into the handover of patients and its implications for the safety of patients, Eur. J. Med. Health Sci. 3(2), 27-34.

https://doi.org/10.34104/ejmhs.021.027034

2) Ahmed A, Senousy $T$ El, Gomaa N, and Rahman AA. (2017). Quality Improvement in Central Sterile Supply Department: Educational Interventions, IOSR J. of Nursing and Health Sci. 6(2), 06-16.

https://iosrjournals.org/iosr-jnhs/papers/vol6-issue 2/Version-9/B0602090616.pdf

3) Damani, N.N. (2019). Manual of infection prevention and control. Oxford; New York, NY: Oxford University Press.

https://www.worldcat.org/title/manual-of-infectio n-prevention-and-control/oclc/1084402073

4) Identifying Healthcare-associated Infections (HAI) for NHSN Surveillance, CDC, 2021). Centers for Disease Control and Prevention. Available from -

www.cdc.gov/nhsn/PDFs/pscManual/2PSC_Identif yingHAIs_NHSNcurrent.pdf

5) Kaushal G, Doke P, Shah A, and Verma V. (2015). An Analysis of Knowledge, Attitude and Practices regarding Standard Precautions of Infection Control and Impact of Knowledge and 
Attitude of ICU Nurses on Self-reported Practices of Infection Control, International J. of Research Foundation of Hospital and Healthcare Administration. 3(2), 79-85.

https://doi.org/10.5005/jp-journals-10035-1041

6) Royal College of Dental Surgeons of Ontario, (2012). Guidelines on Infection Prevention and Control in the Dental Office. Ontario: RCDSO. https://www.rcdso.org/en-ca/rcdso-members/runn ing-your-practice/infection-prevention-and-control

7) Shahen MZ, Mahmud S, Rony MH, Sohana SN, Imran MAS, Al Maruf MA, Azim MAA, Islam MM, Islam MR, Uddin ME, and Alam MS. (2019). Effect of Antibiotic Susceptibility and Inhibitory Activity for the Control of Growth and Survival of Microorganisms of Extracts of Calendula officinalis, Eur. J. Med. Health Sci. 1(3), 1-9. https://doi.org/10.34104/ejmhs.0190109

8) Rutala WA, and Weber DJ. (2016). Disinfection and Sterilization in Health Care Facilities: An Overview and Current Issues, Infectious Disease Clinics of North America. 30(3), 609-637. https://doi.org/10.1016/j.idc.2016.04.002

9) Zaman MSU, Ahmed M, Mahboob N, Iqbal H, Afrin S, and Biswas SM. (2021). Awareness, Attitude and Practice on Sterilization among Healthcare Staffs of a Tertiary Hospital in Bangladesh, Eur. J. Med. Health Sci. 3(1), 1218. https://doi.org/10.34104/ejmhs.021.012018

Citation: Zaman SU, Sadia I, Rahman F, Sharmin R, Haque S, and Ferdoush A. (2021). Necessity of education and training of the healthcare staffs performing sterilization and disinfection of surgical instruments. Eur. J. Med. Health Sci., 3(5), 111-115. https://doi.org/10.34104/ejmhs.021.01110115 @) () 